PROCEEDINGS OF THE

AMERICAN MATHEMATICAL SOCIETY

Volume 128, Number 4, Pages 1023-1030

S 0002-9939(99)05430-1

Article electronically published on August 3, 1999

\title{
ON FOURTH-ORDER ELLIPTIC BOUNDARY VALUE PROBLEMS
}

\author{
C. V. PAO
}

(Communicated by Hal L. Smith)

\begin{abstract}
This paper is concerned with the existence and uniqueness of a solution for a class of fourth-order elliptic boundary value problems. The existence of a solution is proven by the method of upper and lower solutions without any monotone nondecreasing or nonincreasing property of the nonlinear function. Sufficient conditions for the uniqueness of a solution and some techniques for the construction of upper and lower solutions are given. All the existence and uniqueness results are directly applicable to fourth-order two-point boundary value problems.
\end{abstract}

\section{INTRODUCTION}

Fourth-order two-point boundary value problems have been investigated by many researchers in recent years, and various forms of the equation and boundary condition have been discussed (cf. [1]-[3], [5]-[9], [16], [19], [20]). Of special interest is the following fourth-order two-point boundary value problem:

$$
\begin{gathered}
u^{(\mathrm{iv})}=f\left(x, u, u^{\prime \prime}\right) \\
\beta_{0} u(0)-\alpha_{0} u^{\prime}(0)=h_{1}, \quad \beta_{1} u(1)+\alpha_{1} u^{\prime}(1)=h_{1}^{*}, \\
\beta_{0}^{*} u^{\prime \prime}(0)-\alpha_{0}^{*} u^{\prime \prime \prime}(0)=h_{2}, \quad \beta_{1}^{*} u^{\prime \prime}(1)+\alpha_{1}^{*} u^{\prime \prime \prime}(1)=h_{2}^{*} .
\end{gathered}
$$

The above problem arises from the study of static deflection of an elastic bending beam where $u$ denotes the deflection of the beam and $f\left(x, u, u^{\prime \prime}\right)$ represents the loading force that may depend on the deflection and the curvature of the beam. In this paper we extend the problem (0.1) to a more general fourth-order elliptic boundary value problem in the form

$$
\begin{gathered}
\Delta(a(x) \Delta u)=f(x, u, \Delta u) \quad(x \in \Omega), \\
\alpha_{1}(x) \frac{\partial u}{\partial \nu}+\beta_{1}(x) u=h_{1}(x), \quad \alpha_{2}(x) \frac{\partial(a \Delta u)}{\partial \nu}+\beta_{2}(x)(a \Delta u)=h_{2}(x) \quad(x \in \partial \Omega),
\end{gathered}
$$

where $\Omega$ is a smooth bounded domain in $\mathbb{R}^{n}$ with boundary $\partial \Omega, \Delta$ is the Laplace operator in $\Omega$, and $\partial / \partial \nu$ denotes the outward normal derivative on $\partial \Omega$. We assume that $a(x)$ is a positive $C^{1}$-function on $\bar{\Omega} \equiv \Omega \cup \partial \Omega, f(x, u, v)$ is a $C^{\alpha}$-function of its arguments, and for each $i=1,2, \alpha_{i}, \beta_{i}$ and $h_{i}$ are $C^{1+\alpha}$-functions on $\partial \Omega$ with

Received by the editors May 15, 1998.

1991 Mathematics Subject Classification. Primary 35J40, 35J65; Secondary 34B15.

Key words and phrases. Fourth-order elliptic equation, two-point boundary problem, existence-uniqueness, method of upper and lower solutions. 
either $\alpha_{i}=0, \beta_{i}=1$ (Dirichlet condition) or $\alpha_{i}>0, \beta_{i} \geq 0$ (Neumann and Robin condition). As in problem (0.1) the consideration of (1.1) also has an implication in the static deflection of an elastic plate.

Literature dealing with the two-point boundary value problem (0.1) is extensive, and much of the discussion is devoted to the Dirichlet boundary condition

$$
u(0)=h_{1}, \quad u(1)=h_{1}^{*}, \quad u^{\prime \prime}(0)=h_{2}, \quad u^{\prime \prime}(1)=h_{2}^{*} .
$$

An earlier existence result is given in [1] where the function $f(x, u, v)$ is assumed uniformly bounded. This result was extended by Yang [20] who assumed that $f(x, u, v)$ satisfies the growth condition

$$
|f(x, u, v)| \leq a|u|+b|v|+c \text { for } u, v \in \mathbb{R},
$$

where $a, b$ and $c$ are positive constants with $a / \pi^{4}+b / \pi^{2}<1$. In the special case $f(x, u, v)=-c(x) u+d(x)$, Usmani [19] showed that problem (0.1), $(0.1)_{b}$ has a unique solution if $\inf [c(x)]<\pi^{4}$. More recently, Ma, Zhang and $\mathrm{Fu}[9$ obtained an existence result for (0.1), $(0.1)_{b}$ by the method of upper and lower solutions. However, they require that $f(\cdot, u, v)$ be nondecreasing in $u$ and nonincreasing in $v$. Additional works dealing with the existence problem of fourth-order two-point boundary value problems can be found in [2, 3], [5]-[9], [16].

In the case of the elliptic boundary value problem (1.1) the existence problem was investigated by many researchers in the framework of coupled system of second-order equations (e.g. see [12, 13, 15, 18]). Cosner and Schaefer [4] studied a comparison principle for a class of fourth-order elliptic operators, while Tarantello [17] showed the existence of a negative solution for the problem

$$
\begin{array}{ll}
\Delta^{2} u+c \Delta u=b\left[(u+1)^{+}-1\right] & \text { in } \Omega, \\
u=0, \Delta u=0 & \text { on } \partial \Omega,
\end{array}
$$

where $w^{+} \equiv \max \{w, 0\}$, and $b$ and $c$ are positive constants with $c<\lambda_{0}$ and $b \geq \lambda_{0}\left(\lambda_{0}-c\right)$ (see Eq. (2.6)). More recently, Micheletti and Pistoia [10, 11] investigated the existence of multiple solutions for problem (1.2) and a more general nonlinear function $f(x, u)$ using a variational approach.

The purpose of this paper is to show the existence and uniqueness of a solution to the problem (1.1), including the two-point boundary value problem (0.1), by the method of upper and lower solutions without any monotone condition on $f(x, u, v)$. When $f(x, u, v)$ does have a monotone nondecreasing property in $u$ (but not necessarily nonincreasing in $v$ ) we can construct a sequence which converges monotonically to a maximal solution or a minimal solution, depending on whether the initial iteration is an upper solution or a lower solution (see Theorem 1). It is also shown that under some additional conditions the monotone sequences converge to a unique solution of (1.1) when $f(x, u, v)$ is either nondecreasing or nonincreasing in $u$ (see Theorems 2 and (3). The above existence-uniqueness results, including some techniques for the construction of upper and lower solutions, are stated in section 2. Proofs of these results are given in section 3 .

\section{The MAIN RESUlts}

The existence and uniqueness of a solution to problem (1.1) are obtained in relation to a pair of upper and lower solutions which are defined in the following definition. For notational convenience we set

$$
B_{i}[w] \equiv \alpha_{i} \partial w / \partial \nu+\beta_{i} w, \quad i=1,2 .
$$


Definition 2.1. A pair of functions $\tilde{u}, \hat{u} \in C^{4}(\Omega) \cap C^{2+\alpha}(\bar{\Omega})$ are called coupled upper and lower solutions of (1.1) if $\tilde{u} \geq \hat{u}, \Delta \tilde{u} \leq \Delta \hat{u}$ and

$$
\begin{gathered}
\Delta(a \Delta \tilde{u}) \geq f(x, u, \Delta \tilde{u}), \quad \Delta(a \Delta \hat{u}) \leq f(x, u, \Delta \hat{u}) \quad \text { for } \hat{u} \leq u \leq \tilde{u}, \\
B_{1}[\tilde{u}] \geq h_{1} \geq B_{1}[\hat{u}], \quad B_{2}[a \Delta \tilde{u}] \leq h_{2} \leq B_{2}[a \Delta \hat{u}] .
\end{gathered}
$$

For a given pair of coupled upper and lower solutions $\tilde{u}, \hat{u}$ we define

$$
\langle\hat{u}, \tilde{u}\rangle \equiv\left\{u \in C^{2}(\bar{\Omega}) ; \hat{u} \leq u \leq \tilde{u}, \Delta \tilde{u} \leq \Delta u \leq \Delta \hat{u}\right\}
$$

and make the following basic hypothesis.

$\left(H_{1}\right): f(x, u, v)$ is Hölder continuous in $x$ and satisfies the Lipschitz condition

$$
\begin{array}{r}
\left|f\left(x, u_{1}, v_{1}\right)-f\left(x, u_{2}, v_{2}\right)\right| \leq K\left(\left|u_{1}-u_{2}\right|+\left|v_{1}-v_{2}\right|\right) \quad(x \in \bar{\Omega}), \\
\text { for } \hat{u} \leq u_{j} \leq \tilde{u}, \Delta \tilde{u} \leq v_{j} \leq \Delta \hat{u} \quad(j=1,2),
\end{array}
$$

where $K$ is a positive constant.

For the existence of maximal and minimal solutions we also assume that $f(\cdot, u, v)$ possesses the following monotone nondecreasing property in $u$ :

$$
f\left(x, u_{1}, v\right) \leq f\left(x, u_{2}, v\right) \text { for } \hat{u} \leq u_{1} \leq u_{2} \leq \tilde{u}, \Delta \tilde{u} \leq v \leq \Delta \hat{u} .
$$

Under this condition the differential inequalities for $\tilde{u}$ and $\hat{u}$ in (2.1) become

$$
\Delta(a \Delta \tilde{u}) \geq f(x, \tilde{u}, \Delta \tilde{u}), \quad \Delta(a \Delta \hat{u}) \leq f(x, \hat{u}, \Delta \hat{u}) .
$$

Theorem 1. Let $\tilde{u}, \hat{u}$ be a pair of coupled upper and lower solutions, and let Hypothesis $\left(H_{1}\right)$ hold. Then there exists at least one solution $u^{*} \in\langle\hat{u}, \tilde{u}\rangle$ to (1.1). If, in addition, $\left(H_{2}\right)$ holds, then problem (1.1) has a maximal solution $\bar{u}$ and a minimal solution $\underline{u}$ such that $\hat{u} \leq \underline{u} \leq \bar{u} \leq \tilde{u}$. In fact, there exist sequences $\left\{\bar{u}^{(k)}\right\},\left\{\underline{u}^{(k)}\right\}$ with $\bar{u}^{(0)}=\tilde{u}, \underline{u}^{(0)}=\hat{u}$ such that $\left\{\bar{u}^{(k)}\right\}$ converges monotonically from above to $\bar{u}$ and $\left\{\underline{u}^{(k)}\right\}$ converges monotonically from below to $\underline{u}$.

We recall that the maximal and minimal solutions in Theorem 1 are in the sense that if $u$ is a solution in $\langle\hat{u}, \tilde{u}\rangle$, then $\underline{u} \leq u \leq \bar{u}$. Hence problem (1.1) has a unique solution in $\langle\hat{u}, \tilde{u}\rangle$ if $\bar{u}=\underline{u}$. To ensure this we assume that $f(\cdot, u, v)$ is a $C^{1}$-function and $B_{1}=B_{2} \equiv B$ (that is, $\left.\alpha_{1}=\alpha_{2} \equiv \alpha, \beta_{1}=\beta_{2} \equiv \beta\right)$.

Let $\lambda_{0}$ be the smallest eigenvalue and $\phi$ the corresponding positive eigenfunction of the eigenvalue problem

$$
\Delta \phi^{\prime \prime}+\lambda \phi=0 \quad \text { in } \Omega, \quad B[\phi]=0 \quad \text { on } \partial \Omega .
$$

It is well-known that $\lambda_{0}>0$ when $\beta(x) \not \equiv 0$, and $\lambda_{0}=0$ when $\beta(x) \equiv 0$. Define

$$
\begin{aligned}
\bar{a} \equiv \max _{(x \in \overline{\bar{\Omega}})}[a(x)], & \underline{a} \equiv \min _{(x \in \overline{\bar{\Omega}})}[a(x)], \\
M_{1} \equiv \max _{(x, u, v) \in Q}\left|f_{u}(x, u, v)\right|, & m_{1}=\min _{(x, u, v) \in Q}\left|f_{u}(x, u, v)\right|, \\
M_{2} \equiv \max _{(x, u, v) \in Q}\left[-a^{-1}(x) f_{v}(x, u, v)\right], & m_{2}=\min _{(x, u, v) \in Q}\left[-a^{-1}(x) f_{v}(x, u, v)\right],
\end{aligned}
$$

where $Q \equiv\{(x, u, v) ; x \in \bar{\Omega}, \hat{u} \leq u \leq \tilde{u}, \Delta \tilde{u} \leq v \leq \Delta \hat{u}\}$. Then we have the following uniqueness result. 
Theorem 2. Let the conditions in Theorem 1 be satisfied, and let $B_{1}=B_{2} \equiv B$ and $f(\cdot, u, v)$ be a $C^{1}$-function of $(u, v)$. If either

$$
\lambda_{0}\left(\lambda_{0}-M_{2}\right)>M_{1} / \underline{a} \text { or } \lambda_{0}\left(\lambda_{0}-m_{2}\right)<m_{1} / \bar{a},
$$

then problem 1.1 has a unique solution $u^{*} \in\langle\hat{u}, \tilde{u}\rangle$.

The existence-uniqueness result in Theorem 2 remains true if the monotone nondecreasing property (2.4) is replaced by the following monotone nonincreasing property.

$\left(H_{2}\right)^{\prime}$ :

$$
f\left(x, u_{1}, v\right) \geq f\left(x, u_{2}, v\right) \quad \text { for } \hat{u} \leq u_{1} \leq u_{2} \leq \tilde{u}, \Delta \tilde{u} \leq v \leq \Delta \hat{u} .
$$

In this situation the differential inequalities in (2.1) for $\tilde{u}$ and $\hat{u}$ are reduced to

$$
\Delta(a \Delta \tilde{u}) \geq f(x, \hat{u}, \Delta \tilde{u}), \quad \Delta(a \Delta \hat{u}) \leq f(x, \tilde{u}, \Delta \hat{u}),
$$

and we have the following analogous result.

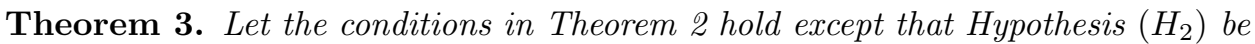
replaced by $\left(H_{2}\right)^{\prime}$. Then problem (1.1) has a unique solution $u^{*} \in\langle\hat{u}, \tilde{u}\rangle$. Moreover, there exist sequences $\left\{\bar{u}^{(k)}\right\},\left\{\underline{u}^{(k)}\right\}$ which converge monotonically to $u^{*}$ as $k \rightarrow \infty$.

Remark 2.1. It is obvious that all the conclusions in Theorems 1 to 3 are directly applicable to the two-point boundary problem (0.1) where $\alpha_{i} \geq 0, \beta_{i} \geq 0$ with $\alpha_{i}+\beta_{i}>0$, and $\alpha_{i}^{*} \geq 0, \beta_{i}^{*} \geq 0$ with $\alpha_{i}^{*}+\beta_{i}^{*}>0(i=0,1)$.

Construction of upper and lower solutions. It is seen from the above theorems that the main condition for the existence problem of (1.1) is the existence of a pair of coupled upper and lower solutions. In the following we give some sufficient conditions on $f(\cdot, u, v)$ and the boundary condition for the construction of these functions.

(a) Suppose $\beta_{1}(x) \not \equiv 0, \beta_{2}(x) \not \equiv 0$, and there exists a constant $M>0$ such that

$$
|f(x, u, v)| \leq M \quad \text { for } u, v \in \mathbb{R} .
$$

Then for any $h_{1}(x)$ and $h_{2}(x)$, each of the equations

$$
\Delta(a \Delta \bar{U})=M \quad \text { and } \quad \Delta(a \Delta \underline{U})=-M
$$

under the same boundary condition as that in (1.1) has a unique solution $\bar{U}$ and $\underline{U}$, respectively, and $\bar{U} \geq \underline{U}$ and $\Delta \bar{U} \leq \Delta \underline{U}$. It is easy to verify that the pair $\widetilde{u}=\bar{U}$ and $\hat{u}=\underline{U}$ are coupled upper and lower solutions of (1.1). This construction ensures the existence of a solution that extends a result of [1] to the more general problem (1.1). that

(b) Suppose $h_{2}(x)=0$ and there exist constants $c_{1}, c_{2}$ with $c_{1} \geq c_{2} \geq 0$ such

$$
\begin{gathered}
c_{1} \beta_{i}(x) \geq h_{i}(x) \geq c_{2} \beta_{i}(x) \quad(i=1,2), \\
f(x, u, 0)=0 \quad \text { for } c_{2} \leq u \leq c_{1} \quad(x \in \Omega) .
\end{gathered}
$$

Then $\tilde{u}=c_{1}$ and $\hat{u}=c_{2}$ are coupled upper and lower solutions. that

(c) Suppose $B_{1}=B_{2}, h_{1}(x)=h_{2}(x)$, and there exists a constant $M \geq 0$ such

$$
f(x, u, 0) \geq 0, \quad f\left(x, u,-\lambda_{0} v\right) \leq \lambda_{0}^{2} v \quad \text { for } u, v \in[0, M]
$$


where $\lambda_{0}$ is the smallest eigenvalue of (2.6) $)$. It is easy to verify that the pair $\tilde{u}=M \phi$ and $\hat{u}=0$ are coupled upper and lower solutions, where $\phi$ is the normalized positive eigenfunction corresponding to $\lambda_{0}$.

(d) Consider the two-point boundary value problem (0.1) with the boundary condition $(0.1)_{b}$ Assume that $h_{1}(x) \geq 0, h_{2}(x) \leq 0$ and there exist positive constants $a, b$ and $c$ with $a / \pi^{4}+b / \pi^{2}<1$ such that

$$
\begin{array}{r}
f(x, 0,0) \geq 0, \quad f_{u}(x, u, v) \geq 0, \quad f(x, u,-v) \leq a u+b v+c \\
\text { for } u \geq 0, v \geq 0 \quad(0 \leq x \leq 1) .
\end{array}
$$

It is easy to verify that by choosing a sufficiently small $\delta>0$ and a sufficiently large $\rho>0$ the pair $\tilde{u}=\rho \sin \gamma(x+\delta)$ and $\hat{u}=0$ are upper and lower solutions, where $\gamma=\pi(1+2 \delta)$. If, in addition,

$$
f_{u}(x, u, v) \leq a, \quad f_{v}(x, u, v) \leq b \quad \text { for } u \geq 0, v \geq 0(0 \leq x \leq 1),
$$

then condition (2.8) is satisfied with $M_{1}=a, M_{2}=b$, and by Theorem 2 problem (0.1), $(0.1)_{b}$ has a unique nonnegative solution $u^{*}$. By the maximum principle, $u^{*}$ is the unique positive solution if either $f(x, 0,0) \neq 0$ or $h_{1}+h_{1}^{*} \neq 0$ or $h_{2}+h_{2}^{*} \neq 0$ (cf. 12, 14).

(e) Consider the problem (1.2) where $c \leq \lambda_{0}$ and $b \geq 0$. Denote by $\phi^{*}(x)$ the positive eigenfunction corresponding to the smallest eigenvalue of (2.6) in a larger domain $\Omega^{*} \supset \bar{\Omega}$. This implies that for some constant $\varepsilon_{0}>0, \phi^{*}(x) \geq \varepsilon_{0}$ on $\bar{\Omega}$. It is easy to verify that for a sufficiently small constant $\delta>0$ and a sufficiently large constant $\rho>0$, the pair $\tilde{u}=-\delta \phi$ and $\hat{u}=-\rho \phi^{*}$ are coupled upper and lower solutions of (1.2) if

$$
b \geq \lambda_{0}\left(\lambda_{0}-c\right) .
$$

This implies that under condition (2.16) problem (1.2) has at least one negative solution, a result obtained in [17.

On the other hand, if $b \leq \lambda_{0}\left(\lambda_{0}-c\right)$, then for any constant $\rho_{0}>0$ the pair $\tilde{u}=\rho_{0} \phi$ and $\hat{u}=0$ are coupled upper and lower solutions. Since $f_{u}(u, v)=b$, $f_{v}(u, v)=-c$ for all $u \geq 0$ and $v \in \mathbb{R}$, where $f(u, v)=b\left[(u+1)^{+}-1\right]-c v$, we see that $M_{1}=m_{1}=b$ and $M_{2}=m_{2}=|c|$, and therefore condition (2.8) holds if $\lambda_{0}\left(\lambda_{0}-|c|\right) \neq b$. By the uniqueness result of Theorem [2, $u^{*}=0$ is the only nonnegative solution of (1.2) if

$$
b<\lambda_{0}\left(\lambda_{0}-|c|\right) .
$$

It is interesting to note that if $b=\lambda_{0}\left(\lambda_{0}-c\right)$, then problem (1.2) has an infinite number of solutions (positive and negative) in the form $u=\sigma \phi$, where $\sigma \geq-1$ is an arbitrary constant.

\section{Proof of the theorems}

Let $C^{m+\alpha}(\bar{\Omega})$ be the set of functions in $C^{m}(\bar{\Omega})$ that are Hölder continuous in $\bar{\Omega}$ with exponent $\alpha \in(0,1)$, where $m$ is a nonnegative integer. The set of vector functions $\mathbf{u} \equiv(u, v)$ with $u, v$ in $C^{m+\alpha}(\bar{\Omega})$ is denoted by $\mathcal{C}^{m+\alpha}(\bar{\Omega})$. Let $\tilde{u}, \hat{u}$ be a pair of coupled upper and lower solutions, and let $\tilde{\mathbf{u}} \equiv(\tilde{u},-\Delta \tilde{u}), \hat{\mathbf{u}} \equiv(\hat{u},-\Delta \hat{u})$. Define

$$
\langle\hat{\mathbf{u}}, \tilde{\mathbf{u}}\rangle \equiv\left\{(u, v) \in \mathcal{C}^{2+\alpha}(\bar{\Omega}) ;(\hat{u},-\Delta \hat{u}) \leq(u, v) \leq(\tilde{u},-\Delta \tilde{u})\right\}
$$


By letting $v=-a \Delta u$ we may write (1.1) in the equivalent form

$$
\begin{gathered}
-a \Delta u=v, \quad B_{1}[u]=h_{1}, \\
-\Delta v=f(x, u,-v / a), \quad B_{2}[v]=-h_{2} .
\end{gathered}
$$

To prove the existence and uniqueness of a solution to (1.1) it suffices to show the same for (3.2).

Proof of Theorem 1. Let $\tilde{v}=-a \Delta \tilde{u}, \hat{v}=-a \Delta \hat{u}$. It is easy to see from (2.1) that the pair $(\tilde{u}, \tilde{v}),(\hat{u}, \hat{v})$ satisfy the relation

$$
\begin{gathered}
-a \Delta \tilde{u} \geq v, \quad-\Delta \tilde{v} \geq f(x, u,-\tilde{v} / a) \\
-a \Delta \hat{v} \leq v, \quad-\Delta \hat{v} \leq f(x, u,-\hat{v} / a) \quad \text { for } \hat{u} \leq u \leq \tilde{u}, \hat{v} \leq v \leq \tilde{v} \quad(x \in \Omega), \\
B_{1}[\tilde{u}] \geq h_{1}(x) \geq B_{1}[\hat{u}], \quad B_{2}[\tilde{v}] \geq-h_{2}(x) \geq B_{2}[\hat{v}] \quad(x \in \partial \Omega) .
\end{gathered}
$$

This implies that the pair $(\tilde{u}, \tilde{v}),(\hat{u}, \hat{v})$ are "generalized" coupled upper and lower solutions of (3.2) in the sense of [12]. The existence of a solution to (3.2) follows from Theorem 8.10.5 of [12] (see also [15, 18]). When condition (2.4) is satisfied the vector function

$$
\mathbf{f}(x, u, v) \equiv(v, f(x, u,-v / a))
$$

is quasimonotone nondecreasing in $\langle\hat{u}, \tilde{u}\rangle$. The existence of the maximal solution $\bar{u}$ and the minimal solution $\underline{u}$ and the convergence of the monotone sequences $\left\{\bar{u}^{(k)}\right\}$ and $\left\{\underline{u}^{(k)}\right\}$ follow from Theorem 8.4.1 of [12].

Proof of Theorem 圆. It suffices to show that $\bar{u}=\underline{u}$ where $\bar{u}$ and $\underline{u}$ are the respective maximal and minimal solutions of (1.1). Let $w=\bar{u}-\underline{u}, z=\bar{v}-\underline{v}$, where $\bar{v}=-a \Delta \bar{u}$, $\underline{v}=-a \Delta \underline{u}$. By (3.2) and the maximal and minimal property of $(\bar{u}, \bar{v})$ and $(\underline{u}, \underline{v})$, we have $\bar{w} \geq 0, z \geq 0$ and

$$
\begin{gathered}
-a \Delta w=z, \quad-\Delta z=f(x, \bar{u},-\bar{v} / a)-f(x, \underline{u},-\underline{v} / a) \quad(x \in \Omega), \\
B_{1}[w]=B_{2}[z]=0 \quad(x \in \partial \Omega) .
\end{gathered}
$$

Multiplying the differential equations by $\phi$, integrating over $\Omega$, and applying the mean-value theorem lead to

$$
\begin{aligned}
& -\int_{\Omega} \phi \Delta w d x=\int_{\Omega}(\phi z / a) d x \\
& -\int_{\Omega} \phi \Delta z d x=\int_{\Omega} \phi\left[f_{u}\left(x, \xi_{1}, \xi_{2}\right) w-f_{v}\left(x, \xi_{1}, \xi_{2}\right) z / a\right] d x,
\end{aligned}
$$

where $\xi_{1} \equiv \xi_{1}(x)$ and $\xi_{2} \equiv \xi_{2}(x)$ are some intermediate values. By the Green's theorem and using (2.6), (2.7) and the boundary condition in (3.4) we obtain the relation

$$
\begin{gathered}
\lambda_{0} \int_{\Omega} \phi w d x=\int_{\Omega}(\phi z / a) d x \\
\int_{\Omega}\left(M_{1} \phi w+M_{2} \phi z\right) d x \geq \lambda_{0} \int_{\Omega} \phi z d x \geq \int_{\Omega}\left(m_{1} \phi w+m_{2} \phi z\right) d x .
\end{gathered}
$$

This leads to the relation

$$
\begin{gathered}
\underline{a}^{-1} \int_{\Omega} \phi z d x \geq \int_{\Omega}(\phi z / a) d x=\lambda_{0} \int_{\Omega} \phi w d x \geq \bar{a}^{-1} \int_{\Omega} \phi z d x, \\
M_{1}\left(\lambda_{0}-M_{2}\right)^{-1} \int_{\Omega} \phi w d x \geq \int_{\Omega} \phi z d x \geq m_{1}\left(\lambda_{0}-m_{2}\right)^{-1} \int_{\Omega} \phi w d x
\end{gathered}
$$


which yields

$$
M_{1}\left[\underline{a} \lambda_{0}\left(\lambda_{0}-M_{2}\right)\right]^{-1} \int_{\Omega} \phi w d x \geq \int_{\Omega} \phi w d x \geq m_{1}\left[\bar{a} \lambda_{0}\left(\lambda_{0}-m_{2}\right)\right]^{-1} \int_{\Omega} \phi w d x .
$$

Hence under either one of the conditions in (2.8) the above relation can hold only when $w=0$. This shows that $\bar{u}=\underline{u}$ and is the unique solution of (1.1).

Proof of Theorem 3 . When Hypothesis $\left(H_{2}\right)$ is replaced by $\left(H_{2}\right)^{\prime}$ the function $\mathbf{f}(x, u, v)$ in (3.3) is mixed quasimonotone in $\langle\hat{u}, \tilde{u}\rangle$. By Theorem 8.10.1 of [12] there exist sequences $\left\{\bar{u}^{(k)}, \bar{v}^{(k)}\right\},\left\{\underline{u}^{(k)}, \underline{v}^{(k)}\right\}$ which converge to a pair of quasisolutions $(\bar{u}, \bar{v})$ and $(\underline{u}, \underline{v})$ that satisfy the relation

$$
\begin{gathered}
-a \Delta \bar{u}=\bar{v}, \quad-\Delta \bar{v}-f(x, \underline{u},-\bar{v} / a) \quad(x \in \Omega), \\
-\Delta \underline{u}=\underline{v}, \quad-\Delta \underline{v}=f(x, \bar{u},-\underline{v} / a) \quad\left(x \in{ }^{2} \quad\right. \\
B_{1}[\bar{u}]=B_{1}[\underline{u}]=h_{1}(x), \quad B_{2}[\bar{v}]=B_{2}[\underline{v}]=-h_{2}(x) \quad(x \in \partial \Omega) .
\end{gathered}
$$

To guarantee that this pair of quasisolutions are true solutions and yield a unique solution it suffices to show $(\bar{u}, \bar{v})-(\underline{u}, \underline{v})$ (cf. [12]). However, since the pair $w \equiv \bar{u}-\underline{u}$ and $z \equiv \bar{v}-\underline{v}$ satisfy the relation (3.4) except with the equation for $z$ replaced by

$$
-\Delta z=f(x, \underline{u},-\bar{v} / a)-f(x, \bar{u},-\underline{v} / a),
$$

we conclude from the argument in the proof of Theorem 2 that $\bar{u}=\underline{u}$ and $\bar{v}=\underline{v}$. This proves the theorem.

\section{ACKNOWLEDGEMENT}

The author would like to thank the referee for his/her suggestions which lead to the present more general problem (1.1).

\section{REFERENCES}

1. A. R. Aftabizadeh, Existence and uniqueness theorems for fourth-order boundary value problems, J. Math. Anal. Appl. 116 (1986), 415-426. MR 87g:34017]

2. R. Agarwal, On fourth-order boundary value problems arising in beam analysis, Differential Integral Eqs. 2 (1989), 91-110. MR 89k:34038

3. A. Cabada, The method of lower and upper solutions for second, third, fourth and higher order boundary value problems, J. Math. Anal. Appl. 185 (1994), 302-320. MR 95h:34033

4. C. Cosner and P. W. Schaefer, A comparison principle for a class of fourth order elliptic operators, J. Math. Anal. Appl. 128 (1987), 488-494. MR 88k:35012

5. C. De Coster, C. Fabry and F. Munyamarere, Nonresonance conditions for fourth-order nonlinear boundary value problems, Internat. J. Math. Meth. Sci. 17 (1994), 725-740. MR 95g:34032

6. M. A. Del Pino and R. F. Manasevich, Existence for a fourth-order nonlinear boundary problem under a two-parameter nonresonance condition, Proc. Amer. Math. Soc. 112 (1991), 81-86. MR 91h:34027

7. C. P. Gupta, Existence and uniqueness theorem for the bending of an elastic beam equation, Applicable Anal. 26 (1988), 289-304. MR 89m:34027

8. A. C. Lazer and P. J. McKenna, Global bifurcation and a theorem of Tarantello, J. Math. Anal. Appl. 181 (1994), 648-655. MR 95b:34033

9. R. Y. Ma, J. H. Zhang and S. M. Fu, The method of lower and upper solutions for fourth-order two-point boundary value problems, J. Math. Anal. Appl. 215 (1997), 415-422. MR 98i:34037

10. A. M. Micheletti and A. Pistoia, Multiplicity results for a fourth-order semilinear problem, Nonlinear Analysis 31 (1998), 895-908. MR 99d:35059

11. _ Nontrivial solutions for some fourth-order semilinear elliptic problems, Nonlinear Analysis 34 (1998), 509-523. CMP 98:15

12. C. V. Pao, Nonlinear parabolic and elliptic equations, Plenum Press, New York, 1992. MR 94c:35002 
13. 165-198. MR 83i:35094

14. M. H. Protter and H. F. Weinberger, Maximum principles in differential equations, Prentice Hall, Englewood Cliffs, N.J., 1967. MR 86f:35034 MR 36:2935

15. K. Schmitt, Boundary value problems for quasilinear second order elliptic equations, Nonlinear Analysis 2 (1978), 263-309. MR 80b:35064

16. J. Schroder, Fourth-order two-point boundary value problems; estimate by two side bounds, Nonlinear Analysis 8 (1984), 107-114. MR 85g:65092

17. G. Tarantello, A note on a semilinear elliptic problem, Differential Integral Equations $\mathbf{5}$ (1992), 561-565. MR 93h:35073

18. L. Y. Tsai, Existence of solutions of nonlinear elliptic systems, Bull. Inst. Math. Acad. Sinica 8 (1980), 111-127. MR 81e:35052

19. R. A. Usmani, A uniqueness theorem for a boundary value problem, Proc. Amer. Math. Soc. 77 (1979), 320-335. MR 80j:34018

20. Y. Yang, Fourth-order two-point boundary value problem, Proc. Amer. Math. Soc. 104 (1988), 175-180. MR 89g:34021

Department of Mathematics, North Carolina State University, Raleigh, North CarOLINA 27695-8205

E-mail address: cvpao@eos.ncsu.edu 